\title{
Pharmacokinetic Herb-Drug Interactions: Are Preventive Screenings Necessary and Appropriate?
}

Veronika Butterweck ${ }^{1}$

Hartmut Derendorf'

Wilhelm Gaus ${ }^{2}$

Adolf Nahrstedt ${ }^{3}$

Volker Schulz ${ }^{4}$

Matthias Unger 5

\section{Abstract}

Pharmacokinetic interactions often occur as a result of activity changes of drug-metabolizing and transporting proteins, especially cytochrome P450 (CYP) isoenzymes and P-glycoprotein (P-gp). The activity of these enzymes and drug transporters can be enhanced or inhibited by synthetic drugs as well as by natural products. Since the number of herb-drug interactions has increased in recent years, systematic in vitro screenings and more clinical studies to identify such interactions were proposed for herbal medicinal products. However, previous results regarding this issue are not only contradictory but also of less predictability. One reason for the discrepancies could be the lack of validation of the recommended in vitro tests. Furthermore, it has to be considered that pharmacokinetic drug interactions are not only mediated by herbal medicines but also by several foods, beverages and life-style products. Since herbal medicines are considered to have a broad therapeutic range, a preventive risk assessment for pharmacokinetic drug interactions should first be realized for synthetic drugs with a narrow therapeutic index. Efforts to identify all possible interactions will lead to limitless investigations and to inconsistent decisions.

\section{Key words}

Pharmacokinetic drug interactions · cytochrome P450 • P-glycoprotein $\cdot$ herb-drug interactions $\cdot$ food-drug interactions $\cdot$ preventive screenings

\section{Introduction}

Recently, interactions of herbal medicines with synthetic drugs have come into focus of particular interest. In the past 3 years, more than 50 papers were published regarding interactions between St. John's wort and prescription drugs only (for summary, see [1], [2], [3]). Meanwhile, drug-interactions are presumed for more than 150 herbal medicines; the alphabetical list includes plants from Adonis vernalis to Taxus brevifolia [4], [5]. Depending on the resources consulted, the quality of information regarding herb-drug interactions can vary significantly. A survey of the current herb-drug interaction literature identified a number of alarming articles in both the popular press and medical journals. Most information is based on unvalidated case reports, on reviews, theory, personal opinions or in vitro studies of limited relevance, or has focused primarily on drugs with narrow therapeutic ranges (e.g., digoxin, warfarin) [6]. In these articles, contradictions appear which need to be critically and carefully evaluated, especially with regard to the experimental methods and to the interpretation of the data. These issues further raise the question about evaluation aspects of in vitro drug metabolism studies based on enzyme assays, since results of those studies are often used to predict in vivo herb-drug interactions. The present review tries to differentiate between theoretical herb-drug

Affiliation

${ }^{1}$ Department of Pharmaceutics, University of Florida, Gainesville, FL, USA

${ }^{2}$ Abteilung Biometrie und Medizinische Dokumentation, Ulm, Germany

${ }^{3}$ Institute of Pharmaceutical Biology and Phytochemistry, Münster, Germany

${ }^{4}$ Berlin, Germany

${ }^{5}$ Institut für Pharmazie, Würzburg, Germany

Correspondence

Prof. Dr. med. Volker Schulz · Oranienburger Chaussee 25 · 13465 Berlin · Germany ·

E-mail: v.schulz.berlin@t-online.de

Received June 14, 2004 • Accepted July 12, 2004

Bibliography

Planta Med 2004; 70: 784-791 • c Georg Thieme Verlag KG Stuttgart · New York

DOI $10.1055 / \mathrm{s}-2004-827223$

ISSN 0032-0943 
interactions and those that are of clinical relevance. In addition practical aspects of in vitro drug metabolism studies and the use of in vitro/in vivo correlation approaches described in the literature are critically reviewed and commented. A bibliography [7] regarding interactions between drugs and herbs and between drugs and food-supplements is available at http://www.uniulm.de/uni/fak/medizin/biodok/v4/herb_drug_interactions.html. These webpages provide an outline of more than 150 articles classified by type of study and substances that are involved in the interaction. In these articles results on 45 different substances (herbs and food supplements) are reported. Results are derived from reviews, randomized controlled trials, non-randomized studies, in vitro and animal experiments as well as personal opinions. The evaluated articles were published between January 1989 and June 2004. Literature was retrieved by extended searches in reference databanks. Furthermore, we received articles from the German drug authority BfArM and via personal communications, especially with members of the BfArM's Commission E, responsible for phytotherapy. The bibliography contains an alphabetical index of first and co-authors as well as an alphabetical index of herbs and food-supplements.

\section{CYP450 and P-Glycoprotein Mediated Interactions}

Most xenobiotics (including phytopharmaceuticals) undergo chemical modifications in the body before they can be effectively eliminated. Biotransformation is the process by which usually lipophilic drugs are rendered more hydrophilic and, hence, more easily excreted. The metabolic conversion of drugs generally is enzymatic in nature. The cytochrome P450 enzyme family is the major catalyst of phase I drug biotransformation reactions. CYP enzymes are bound to membranes of the endoplasmatic reticulum and are predominantly expressed in the liver, although they are also present in extrahepatic tissues such as the gut mucosa [8]. In humans, 16 gene families and 29 subfamilies have been identified to date. CYP3A4 is the most abundantly expressed isoform and represents approximately $30-40 \%$ of the total CYP protein in human adult liver [8]. The isoenzymes most commonly involved in drug metabolism are CYP3A4, CYP2D6 and CYP1A2 [9].

Striking overlaps of substrates and inhibitors between CYP3A4 and P-glycoprotein (P-gp) were reported by Wacher et al. [10]. $\mathrm{P}$-gp is a transmembrane protein of $170-180 \mathrm{kDa}$ and acts as a multidrug resistance factor in tumor cells, thereby reducing the intracellular accumulation of drugs. P-gp, an ATP-dependent primary active transporter belonging to the $A B C$ transporter superfamily, exists not only in tumor cells but also in plasma membranes of many normal tissues, where it serves as an efflux transporter of xenobiotics [11]. In the intestine, P-gp is located at the apical surface of epithelial cells and interferes with drug absorption by pumping out a variety of orally administered drugs into the intestinal lumen [12]. After uptake by the enterocyte, many lipophilic drugs are either metabolized by CYP3A4 or pumped back into the lumen by the P-gp transporter. Therefore, CYP3A4 and P-gp may act in tandem as a barrier to oral delivery of many drugs. However, it has to be considered that the interaction between substrates and CYP enzymes or the P-gp efflux transporter does not always follow simple enzyme kinetics [13]. The pattern of this interaction can be classified into at least three major categories: competitive inhibition, non-competitive inhibition and co-operative stimulation. The situation can be even more complicated if allosteric effects are involved in interactions between substrate and inhibitor. Furthermore, metabolic drug-drug interactions can result in tremendous variability in pharmacokinetics, with changes in drug concentrations of over 10 -fold being reported [14], [15]. Thus, the complexity of the molecular mechanism for those interactions hinders our ability to predict the potential of CYP or P-gp mediated drug-drug, drug-herb or drugfood interaction, either quantitatively or qualitatively.

Since its origin more than 3.5 billion years ago, the CYP450 gene family has diversified to accommodate the metabolism of a growing number of many xeno- and endobiotics. These P450 s play key roles in steroid hormone biosynthesis, the activation and detoxification of many drugs and environmentally contaminating chemicals, the metabolism of polyunsaturated fatty acids (such as arachidonic acid and prostaglandins), activation of vitamins $A$ and $D_{3}$ to biologically active hormones, the synthesis of a vast array of secondary metabolites in plants and insects (e.g., alkaloids, glycosides, polyphenols), the metabolism of contaminating environmental chemicals to toxic and carcinogenic agents and the list goes on and on [16]. Taken together, the CYP enzyme family has always been able to metabolize biotic inputs in a wellbalanced way. However, these enzymes now must work with the simultaneous input of xenobiotics in the form of medicines. During the last decades, medication has become more synthetic and today patients' bodies are being exposed to "cocktails" of xenobiotics. These synthetic drugs post a challenge to the human metabolic system, especially when different substances are administered concomitantly [17].

Recently, herb-drug interactions have received much attention. The most recent interaction involved Hypericum perforatum, Allium sativum and Echinacea purpurea which have been shown to induce or inhibit the activity on the CYP system, possibly interacting with several medications. Yet, it could be argued that the synthetic medications are the source of interference since the CYP system was originally conditioned to work with secondary products of natural origin. It is noteworthy to mention that drug-food interactions are a commonly overlooked aspect in this context. Some foods, minerals, vitamins, spices and beverages have shown inhibiting or inducing activity on CYP450 enzymes or on P-gp [14], [17], [18], [19]. The search for active compounds causing these interactions leads to secondary products (e.g., flavonoids, polyphenols) which are found ubiquitously in the plant kingdom [20], [21], [22]. When compared to daily food intake the amount of those secondary products is relatively low when taken as a herbal medicine. Thus, a critical assessment of possible herb-drug interaction should also include dietary factors.

\section{Reported Herb-Drug Interaction Based on in vitro Assays and Clinical Trials}

The FDA guidance to industry [23] provides suggestions on current approaches to studies in vitro of drug metabolism and interactions. These guidelines recently have been adapted by the Ger- 
man BfArM authorities. The FDA guidance is intended to encourage routine, thorough evaluation of metabolism and interactions in vitro whenever feasible and appropriate. The following experimental models are described in this document to study drug interactions mediated by CYPs or P-gp: subcellular fraction of human liver tissues (microsomes, S9 subcellular fraction), whole cell models (human liver slices, human hepatocytes, Caco-2 cell monolayers), heterologously expressed and purified human drug-metabolizing enzymes (cDNAs). According to the FDA guidelines, interaction studies with CYP enzymes (recommended minimum spectrum: 3A4, 2D6, 2C9, 1A2, 2C19) and Pgp are performed on three levels: identification of the substrate, enzyme induction, enzyme inhibition. To determine if a new drug inhibits/induces a particular CYP enzyme activity, changes in the metabolism of a specific substrate with varying concentrations of the new drug are monitored. In general, the FDA guidance is directed towards a broad class of synthetic drugs: molecules with a molecular weight below $10 \mathrm{kDa}$ [23]. Recently, these screening models were also used for detecting herb-drug interactions. However, practical experience in screening herbal medicines for in vitro drug interactions is limited to several years and until now these models have not been validated for plant extracts. The following examples are chosen from recently published data.

The group of Foster et al. [18] recently screened aliquots from 6 spices, 20 herbal teas, 7 soybean varieties, and 4 pure plant compounds for their ability to affect the in vitro metabolism of marker substrates by cytochrome P450 2C9, 2C19, 2D6 and 3A4 isoforms. All classes of natural products tested, including the spices, the tea infusions, the soy nutraceuticals and the pure phytochemicals examined in this study, showed significant but variable inhibition (50\% inhibition for the spices, $80-100 \%$ inhibition for the teas, $75 \%$ inhibition for the soy extract) of P450mediated metabolism of at least three marker substrates (Table 1). The screenings conducted in this study clearly demonstrate that "positive" results in in vitro tests can be obtained almost with every single herbal extract. Thus, the question arises why "positive" in vitro effects obtained with phytomedicines necessarily should have consequences regarding the admission of those products while the same effects are neglected when they occur for spices, beverages or foods which usually are more commonly consumed.

Table 1 \% Inhibition of human CYP isoforms, $625 \mu \mathrm{g}$ extract $/ \mathrm{mL}$ (after [18])

\begin{tabular}{|c|c|c|c|}
\hline Extracts & CYP $3 A 4$ & CYP 2D6 & CYP $2 C 9$ \\
\hline Artemisia vulgaris (Turkey) & 97 & 100 & 97 \\
\hline Thyme (Spain) & 97 & 96 & 93 \\
\hline Cloves (Sri Lanka) & 94 & 98 & 99 \\
\hline Curcuma (India) & 93 & 49 & 82 \\
\hline Ginger (China) & 94 & 70 & 53 \\
\hline Black Tea (5 varieties) & $77-84$ & $76-93$ & $92-98$ \\
\hline Hypericum perforatum & 64 & 33 & 68 \\
\hline Matricaria chamomilla & 57 & 54 & 61 \\
\hline Piper methysticum & 50 & 25 & 57 \\
\hline Uncaria tomentosa & 57 & 13 & 11 \\
\hline
\end{tabular}

Perhaps one of the most critical experimental variables in CYP interaction studies is the selection of the correct substrate concentration used in the in vitro incubation mixture; an inappropriate substrate concentration can yield results that are not reflective of the in vivo situation. Since only limited information is available regarding the pharmacokinetics and bioavailability of herbal medicines, concentration ranges for in vitro interaction studies have to be chosen more or less randomly. In order to address this approach, Strandell et al. [19] recently tried to develop a rational procedure to screen natural remedies for their CYP-enzyme inhibiting activity in vitro. These authors expressed the inhibitory potency as the volume that a recommended dose could be diluted into to yield a half-maximal inhibition. This unit could be compared to physiological distribution volumes and could aid in a uniform initial risk assessment. For example, an $\mathrm{IC}_{50}$ value of 5 liters/dose unit indicates that one dose unit diluted to approximately the blood volume of the body would result in a concentration of inhibitory substances equivalent to the recorded $\mathrm{IC}_{50}$ of CYP enzyme inhibition [19]. In this assay, extracts of St. John's wort and Valerian root were potent inhibitors of CYP 3A4 and CYP 2C19, whereas preparations of Echinacea or garlic caused no or only a slight inhibition. However, it has been shown in clinical studies with healthy volunteers that Echinacea as well as garlic selectively modulate CYP3A activity and other CYP enzymes [24], [25]. Thus, the adjustment to a virtual in vivo concentration can be used as a screening model but the results from these assays match with clinical findings only in form of a random distribution.

In either case, the propositions for determination of concentrations were therefore not chosen very reasonably. The enteral induction or inhibition of CYP 3A4 and P-gp - both of them are essential in this case - occurs in the apical cell layer of the intestinal mucosa. However, virtual distribution volumes for reflecting systemically acting drugs are not a useful parameter for the in vivo occurring concentrations. These concentrations only depend on which active plant constituents reach the relevant intestinal area metabolically unaltered. Since for most phytopharmaceuticals data about intestinal metabolism and the area of absorption from the small intestine do not exist, a rational approach for the calculation of concentrations for in vitro studies is impossible.

\section{Fundamental Difficulties in the Assessment of Herb-Drug Interactions}

\section{In vitro studies}

The study of herb-drug interactions is extraordinarily complex because plant extracts are multicomponent mixtures which contain several chemical constituents. For the majority of these multicomponent mixtures, the active constituents are often unknown. Furthermore, in most cases general information about the amount of tannins in these extracts is lacking. The majority of in vitro studies used for screening of drug interactions are based on enzyme assays. However, enzymes are proteins and it is well known that tannins precipitate and therefore inactivate proteins. Thus, unspecific effects due to protein-tannin interaction rather than an enzyme inhibition by xenobiotics cannot be excluded. A critical review of the published literature shows that almost every plant extract tested in an in vitro screening as- 
say revealed an interaction with CYP enzymes. The relevance of these studies is therefore questionable, especially when considering the fact that information regarding the amount of tannins is lacking.

For the purpose of high-throughput drug-drug interaction screening, some CYP substrates have been developed specifically to form a fluorescent product to easily monitor the inhibition of metabolism. When these substrates are used to screen plant extracts this might lead to false positive/negative results since herbal extracts often contain natural compounds with an intrinsic fluorescence which might interfere with these assays. Specifically, a natural product's intrinsic fluorescence might mask enzyme inhibition; whereas its quenching might mimic or simulate inhibition [22], [26]. In a recent study, Zou et al. [25] evaluated the effects of intrinsic fluorescence and quenching in fluorescencebased enzyme inhibition assays of 25 components of popular herbal products. In this study, quercetin had the greatest effect and quenched the fluorescent products of CYP 2D6, 2C19/1A2, and $3 \mathrm{~A} 4 / 2 \mathrm{C} 9$ by 33,55 , and $15 \%$, respectively, thus mimicking a weak inhibition. On the other hand, the intrinsic fluorescence of yangonin exceeded the signal of the fluorescent products by $92-$ $424 \%$, simulating a false negative effect [26].

Since fluorescence and quenching are well known to users of these assays and are discussed within some publications describing fluorimetric assays [27], it would be prudent to include an evaluation of these properties in fluorimetric assay protocols when natural products are used. As an example, the $\mathrm{IC}_{50}$ value for CYP $3 \mathrm{~A} 4$ determined in fluorimetric assays for hypericin - a compound with an intensive native fluorescence does not correlate with values calculated by LC/MS methods: the $\mathrm{IC}_{50}$ value for hypericin was $330 \mu \mathrm{M}$ in a fluorimetric assay [28], whereas it was at $8.7 \mu \mathrm{M}$ when testosterone was used as a CYP3A4 substrate and the concentration of the metabolite $6 \beta$ hydroxytestosterone was detected using mass spectrometry [29]. Since these differences are remarkable, results obtained in fluorimetric assays should be verified by more appropriate methods [22].

Recently the German BfArM published a guideline for the assessment of pharmacokinetic herb-drug interaction studies [30]. The guideline requires that extract concentrations need to be chosen according to their clinical relevance. However, it has to be considered that in contrast to most synthetic drugs pharmacokinetic data are not available yet for the majority of medicinal plants and that - in particular - it is by far not known which actual concentration is realized in those tissues of the human body that are involved in transportation or metabolization. Thus, an in vitro screening of plant extracts in a clinically relevant concentration is nearly impossible. In vitro evaluations may not always accurately reflect changes in drug-drug interaction observed in vivo. The group of Chang et al. [31] evaluated the drug-drug interaction between fluvoxamine (FLV) and clozapine (CLZ) by in vitro and in vivo methods. In vitro studies were conducted using human hepatic microsomal preparations with standard chemical inhibitors of the CYP450 isoenzyme system. For the in vivo study, nine male schizophrenic patients were administered a single dose of CLZ (50 mg) on two separate occasions with a 2-week FLV treatment of $50 \mathrm{mg}$ twice a day in between each CLZ dose.
CLZ plasma levels were measured by HPLC with UV detection for both in vitro and in vivo studies. In vitro, FLV had only modest effects (8.5\%) on CLZ levels. In schizophrenic patients, FLV resulted in a pronounced increase in CLZ plasma concentrations (AUC from $780 \mathrm{ng} / \mathrm{mL} \rightarrow 2218 \mathrm{ng} / \mathrm{mL}$ ). Hence, in vitro interaction data need to be carefully interpreted, a more critical evaluation of the existing literature seems appropriate.

\section{Experimental animal models of drug metabolism}

An alternative approach to assess CYP mediated drug interactions is the use of animal models. From ethical as well as rational reasons, the selection of an appropriate animal experiment is crucial. On comparing various animal models, species differences become obvious. In general, the most common species used for animal studies are rats. Unfortunately, the rat is not an appropriate model for CYP interaction studies, because the most important human P450 enzyme, CYP3A4, is not induced in rats by the typical CYP3A inducer rifampicin and, more importantly, many prototypical substrates of human CYP3A enzymes such as dihydropyridine calcium channel blockers (e.g., nifedipine) are not metabolized by rat CYP3A1 or by other CYP3A forms [32]. Furthermore, in rabbits, the metabolism of one third of the drugs used in human treatment involves other forms of CYP enzymes, thus excluding rabbit models from interaction studies. In contrast, all main activities typical for human CYP enzymes were found in liver microsomes of pigs, minipigs and monkeys. Hence, if no significant differences are found in the future, these species after careful validation will become the only valid animal models for CYP metabolism studies. However, since pig or monkey models are relatively cost effective, they might be unreasonable for first screening purposes. In order to keep the costs on a reasonable level it would be of advantage to have a reliable rat or mouse model. Since knockout/transgenic strategies have opened an entire new avenue for the selection of drug targets, this powerful tool, once developed, might enable us to use such rodent species for CYP mediated drug interaction studies in the future.

\section{Clinical studies}

Each type of pharmacokinetic interaction alters the drug concentration versus time profile which may or may not be clinically relevant. The meaning of altered drug exposure for selecting a drug and prescribing a dose must be interpreted with great care. A higher or lower systemic drug concentration does not necessarily mean a higher or lower therapeutic effect. This is due to the fact that the relationships between drug concentration and drug action, or intensity of desired and/or undesired effects, are usually not linear [33]. The pharmacodynamics of many drugs follow the so-called $\mathrm{E}_{\max }$ model where the relationship between concentration and effect is not linear but can be represented by a sigmoid curve. With increasing concentrations, the intensity of the effect approaches a maximum $\left(\mathrm{E}_{\max }\right)$. If the intensity of the effect is close to $\mathrm{E}_{\max }$ then changes in the concentration of the drug will not lead to significant changes. Also for lower concentrations changes in drug effects are almost always much less than the corresponding changes in drug concentrations [34]. This means that doubling the drug concentration must not necessarily equate to doubling the drug effects over most of the concentration versus effect curve where the changes in activity are usually much smaller and sometimes not detectable. Understanding the concentration versus effect curve is pi- 
votal in predicting whether a pharmacokinetic interaction is likely to produce a clinically relevant change in response.

Predicting the clinical significance of pharmacokinetic drug interactions is sometimes difficult especially for drugs where there are no robust methods to quantify effects or side effects. There has been recent effort in the United States by the FDA and the Pharmaceutical Research and Manufacturers of America (PhRMA) to establish some general guidelines to help drug companies, prescribers and patients interpret the clinical significance of drug interactions [35], [36]. These are based on the clinical experience from some well-known drug interactions such as the inhibition of CYP 3A4. For this interaction it could be shown that the benzodiazepine midazolam is a reproducible probe that allows quantitative determination of the interaction potential of an enzyme inhibitor. The degree of interaction can be measured in the form of an increase in the area under the curve (AUC) of the midazolam serum concentrations. However, the important question is, how can one interpret these changes in AUC from a clinical standpoint? The FDA proposed recently to classify changes of midazolam AUC of less than two-fold as being 'weak', which is the case observed after midazolam on co-administration with ranitidine, small portions of grapefruit juice, roxithromycin, fentanyl or azithromycin [36]. AUC changes that range from two- to five-fold, which occur after midazolam on co-administration with erythromycin, diltiazem, fluconazole, verapamil, large portions of grapefruit juice and cimetidine, are classified as being 'moderate'. Changes that exceed a five-fold increase in midazolam AUC are labeled as being ‘strong'. Examples of strong midazolam interactions include those with ketoconazole, itraconazole, mibefradil, clarithromycin or nefazodone. This FDA proposed classification system for the clinical significance of drug interactions determined from in vivo studies is identical to that presented by industry [35]. Strong drug interactions are considered clinically significant and result in contraindications or strong warnings on the product label. The clinical significance of moderate inhibitors may include decisions about dose adjustments that should be based on the concentration-effect relationship.

As mentioned in the preceding paragraphs, most of the published work that considers the clinical relevance of drug interactions has focused on enzyme inhibition. There is no classification system analogous to that proposed by the FDA and PhRMA available for enzyme induction. However, following a similar approach one could propose that a less than two-fold change (decrease in midazolam AUC of less than 50\%) could be labeled 'weak' and a larger change as 'strong'. A differentiation between 'moderate' and 'strong' does not seem to be necessary.

In addition, the individual metabolizing capability of patients has to be taken into consideration. Some of these enzymes can be present in different forms, varying with interindividual and interracial factors. As a result of this genetic polymorphism, some individuals are categorized as "poor", "effective", or "ultrarapid" metabolizers. For example, it has been well established that $3-5 \%$ of Caucasians and $15-20 \%$ of Asians are "poor" CYP2C19 metabolizers [37]. Racial and interindividual variations have recently been reported for additional isoenzymes as well
[37], [38]. Genetic variation in the expression of these enzymes is potentially dangerous, especially in the metabolism of drugs with a narrow therapeutic index, such as warfarin, a substrate for CYP 2C9, CYP 2A6, and CYP 2C19. Polymorphism that impairs warfarin metabolism is common, occurring in $34 \%$ of patients [37].

There are also gender-specific differences. Cigarette smoking is known to induce CYP $1 \mathrm{~A} 2$ activity. A study of the interaction of clozapine and smoking revealed that serum levels of the drug were lower only in male smokers whereas female smokers and non-smokers had unchanged serum levels of the drug [38].

However, the proposed FDA and PhRMA classification system should be used only as an initial guidance since each identified drug interaction needs to be evaluated for clinical significance based on the exposure-response or concentration-effect relationship. It is not useful to publish results of studies that find pharmacokinetic drug interactions and do not discuss clinical implications of the results. In the absence of a clinical context, the results are most often merely alarming for practitioners and patients. When pharmacokinetic drug interaction studies are published without clinical interpretation, publicity can lead to lack of treatment compliance for fear of unwanted side effects. This can be of greater clinical significance than the actual drug interaction.

In order to cover the large field of interactions between synthetic drugs and herbs, dietary supplements or foods epidemiologic studies would be particularly suitable. This suggestion - besides an intensified education of health care practitioners in this field - is discussed in a report previously published by the Natural Health Products Directorate (Health Canada) [6]. As shown by a recently published US paper, such surveys could be more targetoriented than experimental and clinical studies [39].

\section{Dietary Effects on Drug Metabolism}

Drug-food interactions are a commonly overlooked aspect in physicians' prescribing practices. Metabolic food-drug interactions occur when consumption of a particular food modulates the activity of a drug-metabolizing enzyme system, resulting in an alteration of the pharmacokinetis of drugs metabolized by that system. A number of these drug interactions have been reported. Foods that contain complex mixtures of phytochemicals, such as fruits, vegetables, herbs, spices and teas, have the greatest potential to induce or inhibit the activity of drug-metabolizing enzymes [14] (Table 2). For example, foods that are rich in vitamin $\mathrm{K}$ - such as cabbage, Brussels sprouts, asparagus, lettuce, spinach, avocado, and liver - can counteract the activity of warfarin [40], [41]. Cruciferous vegetables have also increased CYP $1 \mathrm{~A} 2$ activity, whereas apiaceous vegetables decreased this enzyme activity [42]. Cigarette smoking as well as the consumption of caffeine-containing beverages also can lead to clinical significant interactions [38], [43], [45]. In the late $1980 \mathrm{~s}$, researchers accidentally discovered that grapefruit juice increased blood levels of felodipine dramatically. Since then the list of drugs interacting with grapefruit juice has expanded. The variety of drugs include dihydropyridine calcium channels blockers such as nife- 
Table 2 Reports about dietary effects on drug metabolism and transport in humans (modified after [14])

\begin{tabular}{|c|c|c|c|c|}
\hline Year & References & Dietary component & Drugs & Changes in $A U C, C L, C_{\max }$, UE \\
\hline 1976 & [59] & Charbroiled meat & Phenacetin & $C_{\max } 75 \% \downarrow$ \\
\hline 1979, 84 & [60], [61] & Cruciferous vegetables & Phenacetin & AUC $49 \% \downarrow$ \\
\hline 1982 & {$[62]$} & Cigarette smoking & Chlorpromazine & AUC $20-40 \% \downarrow$ \\
\hline 1989, 91 & [46], [63], [64] & Grapefruit juice & $\begin{array}{l}\text { Cyclosporin, midazolam, } \\
\text { terfenadine, felodipine, } \\
\text { caffeine, theophylline, } \\
\text { clozapine }\end{array}$ & AUC $30-300 \% \uparrow$ \\
\hline 1994 & [65] & Ethanol & Chlorzoxazone & AUC $50 \% \downarrow$ \\
\hline 1996 & [66] & Broccoli & Caffeine & UE $10-40 \% \uparrow$ \\
\hline 1998 & [67] & Watercress & Chlorzoxazone & AUC $50 \% \uparrow$ \\
\hline 2001 & [68] & Seville orange juice & Felodipine & AUC $76 \% \uparrow$ \\
\hline 2001 & [21] & Red wine & Cyclosporine & CL $50 \% \uparrow A U C 30 \% \downarrow$ \\
\hline 2002 & {$[25]$} & Garlic & Saquinavir & AUC $50 \% \downarrow C_{\max } 54 \% \downarrow$ \\
\hline
\end{tabular}

AUC = area under the curve; $\mathbf{C L}=$ clearance; $\mathbf{C m a x}=$ maximum plasma concentration; $\mathbf{U E}=$ urinary excretion .

dipine [46], nisoldipine [47] and nitrendipine [48], verapamil, cyclosporine [49], tacrolimus [50], midazolam [51], triazolam [52], terfenadine [53], diazepam [54], saquinavir [55], ethinylestradiol [56], and caffeine [57]. However, one could assume that these incidences should have prompted authorities as well as manufacturers to systematically investigate the influence of dietary intake on synthetic drugs. However, as shown in Table $\mathbf{2}$, in the last three decades only a limited number of investigations in this area have been performed. In conclusion, daily diet, intake of supplements and life-style products should be seriously taken into consideration when discussing drug interaction issues because it is obvious that these are part of our daily life. One cannot merely tell patients to refrain from nutrition during treatment with medications. The knowledge of how dietary factors can influence drug therapy can be helpful for the translation of experimental results into clinical relevant data.

Unfortunately, this practical approach is often missing. Recently, Strandell et al. [19] investigated several fish oil products for CYP inhibition. The authors found that two of three products tested were potent inhibitors of all three CYP enzymes investigated (CYP2C19, CYP2D6, CYP3A4). Interestingly, the question as to what impact these results would have for our daily nutrition was not discussed by the authors. The fish oils used for that study constitute with an amount of $1-15 \%$ the main ingredient of approximately 20 different fish species, which represent the main contingent of seafood in our latitudes. Based on an estimated per head consumption of $10-20 \mathrm{~kg}$ fish per year every consumer would approximately ingest $100-3000$-fold of the test doses mentioned above [58]. Therefore, why should medicinally used fish oil capsules get a warning label concerning coadministered synthetic drugs while the amount of daily fish oil intake from seafood is completely ignored?

\section{Conclusion}

An investigation of pharmacokinetic interactions between the active compound and other drugs or substances is of particular importance for synthetic drugs with a narrow therapeutic win- dow. If it comes to herbal medicines, we have to take into account that those products have a relatively wide therapeutic window probably because the human CYP system was originally conditioned to work with compounds of natural origin. Thus, instead of blaming the phytopharmaceuticals it can be assumed that the risk emanates from the synthetic drug, especially from those with a narrow therapeutic window. Based on the suggestions of the American Pharmaceutical Association it is proposed by the authors of this article to evaluate all drugs with a narrow therapeutic index, such as warfarin or cyclosporine and others [9].

Results of interaction studies should be discussed in a clear and useful manner to prescribing practitioners and patients. In case of a potential drug interaction with a widely used food or herb, it is more important to provide information about the interaction to the person using the synthetic drug in contrast to informing the wider population that uses of food or herbals. It would not make sense to put a warning label on grapefruit juice, red wine, cabbage, fish or diverse herbal products. This warning label should be on the synthetic drug so that both the patient and the prescriber are aware of this interaction. For example, labeling of cyclosporine (Neoral, Novartis AG) and simvastatin (Zocor, Merck \& Co, Inc, Whitehouse Station, NU) have warnings about grapefruit juice [36].

Considering physicians' prescribing patterns, it becomes obvious that the majority of patients often use more than one drug which is not surprising in view of the demographics of an aging population. In addition to prescription medication, patients often use multiple products to improve their well-being, including vitamins, food supplements, other nutraceuticals and over the counter drugs. Results from several studies indicate that concomitant use of some of these products can result in unwanted effects due to drug interactions. Since not only synthetic drugs but also some foods, spices and beverages and other nutraceuticals can interact with drugs, the number of potential interactions is limitless. Thus, an extensive and area-wide screening of herb-drug interactions is not only impossible but also inappropriate to improve pharmaceutical drug safety. 


\section{References}

${ }^{1}$ Hammerness P, Basch E, Ulbricht C, Barrette EP, Foppa I, Basch S, Bent S, Boon H, Ernst E. St John's wort: a systematic review of adverse effects and drug interactions for the consultation psychiatrist. Psychosomatics 2003; 44: $271-82$

${ }^{2}$ Henderson L, Yue QY, Bergquist C, Gerden B, Arlett P. St John's wort (Hypericum perforatum): drug interactions and clinical outcomes. Br J Clin Pharmacol 2002; 54: 349-56

${ }^{3}$ Schulz V, Johne A. Side effects and drug interactions. In: Müller WE, ed. St. John's Wort and its active principles in depression and anxiety. Milestones in Drug Therapy Basel, Switzerland: Birkhäuser, 2004

${ }^{4}$ Ernst E. Possible interactions between synthetic and herbal medicinal products. Part 1: a systematic review of the indirect evidence. Perfusion 2000; 13: 4-15

${ }^{5}$ Izzo AA, Ernst E. Interactions between herbal medicines and prescribed drugs: a systematic review. Drugs 2001; 61: 2163 - 75

${ }^{6}$ Boon H, Jurgens T. Drug Interactions with Natural Health Products: A Discussion Paper. Health Canada Website, 2002

${ }^{7}$ Hohl K, Gaus W. Bibliography on interactions between drugs and herbs and between drugs and food-supplements. 2004 http://www.uni-ulm.de/uni/fak/medizin/biodok/v4/herb_drug_interactions.html,

${ }^{8}$ Donato MT, Castell JV. Strategies and molecular probes to investigate the role of cytochrome P450 in drug metabolism: focus on in vitro studies. Clin Pharmacokinet 2003; 42: $153-78$

${ }^{9}$ Limon L. Drug interactions. Decreasing the risk. American Pharmaceutical Association Annual Meeting, Washington, DC: 2000

${ }^{10}$ Wacher VJ, Wu CY, Benet LZ. Overlapping substrate and tissue distribution of cytochrome P450 and P-glycoprotein: implications for drug delivery and activity in cancer chemotherapy. Mol Carcinogen 1995; 13: $129-34$

11 Gatmaitan ZC, Arias IM. Structure and function of P-glycoprotein in normal liver and small intestine. Adv Pharmacol 1993; 24: 77 - 97

12 Lown KS, Mayo RR, Leichtman AB, Hsiao HL, Turgeon DK, SchmiedlinRen P, Brown MB, Guo W, Rossi SJ, Benet LZ, Watkins PB. Role of intestinal P-glycoprotein (mdr1) in interpatient variation in the oral bioavailability of cyclosporine. Clin Pharmacol Ther 1997; 62: 246-80

13 Paine MF, Khalighi M, Fisher JM, Shen DD, Kunze KL, Marsh CL, Perkins JD, Thummel KE. Characterization of interintestinal and intraintestinal variations in human CYP3A-dependent metabolism. J Pharmacol Exp Ther 1997; 283: 1552 - 62

${ }^{14}$ Harris RZ, Jang GR, Tsunoda S. Dietary effects on drug metabolism and transport. Clin Pharmacokinet 2003; 42: 1071-88

15 Kivisto KT, Lilja JJ, Backman JT, Neuvonen PJ. Repeated consumption of grapefruit juice considerably increases plasma concentrations of cisapride. Clin Pharmacol Ther 1999; 66: 448 - 53

16 Estabrook RW. A passion for P450 s (remembrances of the early history of research on cytochrome P450). Drug Metab Dispos 2003; 31 : $1461-73$

17 Sorensen JM. Herb-drug, food-drug, nutrient-drug, and drug-drug interactions: mechanisms involved and their medical implications. J Altern Complement Med 2002; 8: 293 - 308

${ }^{18}$ Foster BC, Vandenhoek S, Hana J, Krantis A, Akhtar MH, Bryan M, Budzinski JW, Ramputh A, Arnason JT. In vitro inhibition of human cytochrome P450-mediated metabolism of marker substrates by natural products. Phytomedicine 2003; 10: 334-42

19 Strandell J, Neil A, Carlin G. An approach to the in vitro evaluation of potential for cytochrome P450 enzyme inhibition from herbals and other natural remedies. Phytomedicine 2004; 11: 98 - 104

${ }^{20}$ Lampen A. Interaktion zwischen Metabolismus und Transport von toxisch relevanten Substanzen in der gastrointestinalen Barriere. Biospektrum 2004; 10: 157 -9

${ }^{21}$ Tsunoda SM, Harris RZ, Christians U, Velez RL, Freeman RB, Benet LZ, Warshaw A. Red wine decreases cyclosporine bioavailability. Clin Pharmacol Ther 2001; 70: $462-7$

22 Unger M. Wie Naturstoffe die Bioverfuegbarkeit von Arzneistoffen beeinflussen. Pharm Ztg 2004; 149: 979-86

${ }^{23}$ U.S. Food Administration A. Guidance for industry: Drug metabolism/ Drug interaction studies in the drug development process: Studies in vitro. Rockville, MD: 1997 http://www.fda.gov/cber/cberftp.html,

${ }^{24}$ Gorski JC, Huang SM, Pinto A, Hamman MA, Hilligoss JK, Zaheer NA, Desai M, Miller M, Hall SD. The effect of echinacea (Echinaced purpurea root) on cytochrome P450 activity in vivo. Clin Pharmacol Ther 2004; 75: 89-100
25 Piscitelli SC, Burstein AH, Welden N, Gallicano KD, Falloon J. The effect of garlic supplements on the pharmacokinetics of saquinavir. Clin Infect Dis 2002; $34: 234-8$

${ }^{26}$ Zou L, Harkey MR, Henderson GL. Effects of intrinsic fluorescence and quenching on fluorescence-based screening of natural products. Phytomedicine 2002; 9: 263-7

${ }^{27}$ Bapiro TE, Egnell AC, Hasler JA, Masimirembwa CM. Application of higher throughput screening (HTS) inhibition assays to evaluate the interaction of antiparasitic drugs with cytochrome P450s. Drug Metab Dispos 2001; 29: $30-5$

${ }^{28}$ Budzinski JW, Foster BC, Vandenhoek S, Arnason JT. An in vitro evaluation of human cytochrome P450 3A4 inhibition by selected commercial herbal extracts and tinctures. Phytomedicine 2000; 7: 273-82

${ }^{29}$ Obach RS. Inhibition of human cytochrome P450 enzymes by constituents of St. John's Wort an herbal preparation used in the treatment of depression. J Pharmacol Exp Ther 2000; 294: 88 - 95

${ }^{30}$ BfArM. Bewertung möglicher pharmakokinetischer Arzneimittel-Interaktionen mit Phytopharmaka (Entwurf). 2004 www.bfarm.de/de/ Arzneimittel/bes_therap/am_pflanzl/int_20 040116.pdf,

${ }^{31}$ Chang WH, Augustin B, Lane HY, ZumBrunnen T, Liu HC, Kazmi Y, Jann MW. In-vitro and in-vivo evaluation of the drug-drug interaction between fluvoxamine and clozapine. Psychopharmacology (Berl) 1999; 145: $91-8$

32 Zuber R, Anzenbacherova E, Anzenbacher P. Cytochromes P450 and experimental models of drug metabolism. J Cell Mol Med 2002; 6: 189-98

33 Derendorf H, Hochhaus G. Handbook of Pharmacokinetic-Pharmacodynamic Correlations. Boca Raton, Florida: 1995: 483

${ }^{34}$ Derendorf H, Meibohm B. Modeling of pharmacokinetic/pharmacodynamic (PK/PD) relationships: concenpts and perspectives. Pharm Res 1999; 16: $176-85$

35 Bjornsson TD, Callaghan JT, Einolf HJ, Fischer V, Gan L, Grimm S, Kao J, King SP, Miwa G, Ni L, Kumar G, McLeod J, Obach SR, Roberts S, Roe A, Shah A, Snikeris F, Sullivan JT, Tweedie D, Vega JM, Walsh J, Wrighton SA. The conduct of in vitro and in vivo drug-drug interaction studies: a PhRMA perspective. J Clin Pharmacol 2003; 43: 443-69

${ }^{36}$ Huang SM. Issues and challenges in the evaluation and labeling of drug interaction potentials of NME FDA Clinical Pharmacology Subcommittee Advisory Board Meeting. Washington DC: 2003

${ }^{37}$ Freeman BD, Zehnbauer BA, McGrath S, Borecki I, Buchman TG. Cytochrome P450 polymorphisms are associated with reduced warfarin dose. Surgery 2000; 128: $281-5$

38 Prior TI, Chue PS, Tibbo P, Baker GB. Drug metabolism and atypical antipsychotics. Eur Neuropsychopharmacol 1999; 9: 301 -9

39 Peng CC, Glassman PA, Trilli LE, Hayes-Hunter J, Good CB. Incidence and severity of potential drug-dietary supplement interactions in primary care patients: an exploratory study of 2 outpatient practices. Arch Intern Med 2004; 164: 630-6

${ }^{40}$ Harder S, Thurmann P. Clinically important drug interactions with anticoagulants. An update. Clin Pharmacokinet 1996; 30: 416-44

${ }^{41}$ Vistisen K, Loft S, Poulsen HE. Cytochrome P450 1A2 activity in man measured by caffeine metabolism: effect of smoking, broccoli and exercise. Adv Exp Med Biol 1991; 283: 407-11

42 Lampe JW, King IB, Li S, Grate MT, Barale KV, Chen C, Feng Z, Potter JD. Brassica vegetables increase and apiaceous vegetables decrease cytochrome P450 1A2 activity in humans: changes in caffeine metabolite ratios in response to controlled vegetable diets. Carcinogenesis 2000; 21: $1157-62$

${ }^{43}$ Jeppesen U, Loft S, Poulsen HE, Brsen K. A fluvoxamine-caffeine interaction study. Pharmacogenetics 1996; 6: $213-22$

${ }^{44}$ Monks TJ, Caldwell J, Smith RL. Influence of methylxanthine-containing foods on theophylline metabolism and kinetics. Clin Pharmacol Ther 1979; 26: $513-24$

45 Cone EJ, Fant RV, Henningfield JE. Nicotine and Tobacco. In: Mozayani A, Raymon LP, eds. Handbook of Drug Interactions Totova, New Jersey: Humana Press, 2004: 463-92

46 Bailey DG, Spence JD, Munoz C, Arnold JMO. Interaction of citrus juices with felodipine and nifedipine. Lancet 1991; 337: 268 -9

${ }^{47}$ Bailey DG, Malcolm AO, Munoz C, Spence JD. Grapefruit juice and naringin on nisoldipine pharmacokinetics. Clin Pharmacol Ther 1993; 54: 589-94

48 Soons PA, Vogels BA, Roosenmalen MC, Shoemaker HC, Uchida E, Edgar B, Lundahl J, Cohen AF, Bremer DD. Grapefruit juice and cimetidine inhibit stereoselective metabolism of nitrendipine in humans. Clin Pharmacol Ther 1991; 50: 393-403 
${ }^{49}$ Ducharme MP, Provenzano R, Dehoorne-Smith M, Edwards DJ. Trough concentration of cyclosporine in blood following administration with grapefruit juice. Br J Clin Pharmacol 1993; 36: 457 -9

50 Westveer MK, Farquhar ML, George P, Mayers JT. Co-administration of grapefruit juice increases tacrolimus levels in liver transplant recipients. Ann Meet Am Soc Transplant Physicians 1996; 202: 115

${ }^{51}$ Kupferschmidt HHT, Ha HR, Ziegler WH, Meier PJ, Krahenbuhl S. Interaction between grapefruit juice and midazolam in human. Clin Pharmacol Ther 1995; 58: $20-8$

${ }^{52}$ Hukkinen SK, Varhe A, Olkkola KT, Neuvonen PJ. Plasma concentrations of triazolam are increased by concomitant ingestion of grapefruit juice. Clin Pharmacol Ther 1995; 58: 127 - 31

53 Benton RE, Honig PK, Zamani K, Cantilena LR, Woosley RL. Grapefruit juice alters terfenadine pharmacokinetics, resulting in prolongation of repolarization on the electrocardiogram. Clin Pharmacol Ther 1996; 59: $383-8$

${ }^{54}$ Ozdemir M, Atkan Y, Boydag BS, Cibgi MI, Musmul A. Interaction between grapefruit jucie and diazepam in human. Eur J Drug Metab Pharmacokin 1998; 23: 55-9

${ }^{55}$ Kupferschmidt HHT, Fattinger KE, Ha HR, Follath F, Krahenbuhl S. Grapefruit juice enhances the bioavailability of the HIV protease inhibitor saquinavir in man. Br J Clin Pharmacol 1998; 45: 355 - 9

${ }^{56}$ Weber A, Jager R, Borner A, Klinger G, Vollanth R, Matth K. Can grapefruit juice influence ethinylestradiol bioavailability. Contraception 1996; $53: 41-7$

${ }^{57}$ Fuhr U, Klittch K, Atab AH. Inhibitory effect of grapefruit juice and its bitter principle, naringenin, on CYP1A2 dependent metabolism of caffeine in man. Br J Clin Pharmacol 1993; 35: 431-6

${ }^{58}$ Simopoulos AP, Kifer RR, Martin RE. Health effects of polyunsaturated fatty acids in seafoods. Orlando, San Diego, New York: Academic Press Inc, 1986: $383-401$
${ }^{59}$ Conney AH, Pantuck EJ, Hsiao KC, Garland WA, Anderson KE, Alvares AP, Kappas A. Enhanced phenacetin metabolism in human subjects fed charcoal-broiled beef. Clin Pharmacol Ther 1976; 20: 633-42

60 Pantuck EJ, Pantuck CB, Garland WA, Min BH, Wattenberg LW, Anderson KE, Kappas A, Conney AH. Stimulatory effect of Brussels sprouts and cabbage on human drug metabolism. Clin Pharmacol Ther 1979; 25: $88-95$

${ }^{61}$ Pantuck EJ, Pantuck CB, Anderson KE, Wattenberg LW, Conney AH, Kappas A. Effect of Brussels sprouts and cabbage on drug conjugation. Clin Pharmacol Ther 1984; 35: 161 -9

62 Pantuck EJ, Pantuck CB, Anderson KE, Conney AH, Kappas A. Cigarette smoking and chlorpromazine disposition and actions. Clin Pharmacol Ther 1982; 31: $533-8$

63 Bailey DG, Arnold JM, Munoz C, Spence JD. Grapefruit juice-felodipine interaction: mechanism, predictability, and effect of naringin. Clin Pharmacol Ther 1993; 53: 637-42

${ }^{64}$ Bailey DG, Kreeft JH, Munoz C, Freeman DJ, Bend JR. Grapefruit juicefelodipine interaction: effect of naringin and 6',7'-dihydroxybergamottin in humans. Clin Pharmacol Ther 1998; 64: 248-56

${ }^{65}$ Girre C, Lucas D, Hispard E, Menez C, Dally S, Menez JF. Assessment of cytochrome P4502E1 induction in alcoholic patients by chlorzoxazone pharmacokinetics. Biochem Pharmacol 1994; 47: 1503 - 8

${ }^{66}$ Kall MA, Vang O, Clausen J. Effects of dietary broccoli on human in vivo drug metabolizing enzymes: evaluation of caffeine, oestrone and chlorzoxazone metabolism. Carcinogenesis 1996; 17: 793-9

${ }^{67}$ Leclercq I, Desager JP, Horsmans Y. Inhibition of chlorzoxazone metabolism, a clinical probe for CYP2E1, by a single ingestion of watercress. Clin Pharmacol Ther 1998; 64: $144-9$

${ }^{68}$ Malhotra S, Bailey DG, Paine MF, Watkins PB. Seville orange juice-felodipine interaction: comparison with dilute grapefruit juice and involvement of furocoumarins. Clin Pharmacol Ther 2001; 69: 14-23 